\title{
Associações entre glifosato e herbicidas pós-emergentes para o controle de trapoeraba em soja $\mathbf{R R}^{\circledR 1}$
}

\section{Glyphosate and post emergence herbicides associations for controlling Commelina benghalensis on soybean $\mathrm{RR}^{\circledR}$}

\author{
Sidnei Roberto de Marchi ${ }^{2}$; Daniel Bogorni ${ }^{3}$; Leandro Biazzi ${ }^{4}$; José Ricardo Bellé ${ }^{4}$
}

Resumo - Este trabalho teve o objetivo de avaliar a seletividade e a eficácia da associação do glifosato com herbicidas pós-emergentes no controle de trapoeraba na cultura da soja Roundup Ready ${ }^{\circledR}$. Para tanto, o experimento foi instalado no delineamento de blocos ao caso, com quatro repetições, onde os tratamentos foram constituídos de quatro doses do herbicida cloransulammetil $\left(10,20,30\right.$ e $\left.40 \mathrm{~g} \mathrm{ha}^{-1}\right)$, quatro doses do herbicida clorimurom-etílico $(2,5,5,0,7,5$ e 10,0 g $\mathrm{ha}^{-1}$ ), imazetapir a $100 \mathrm{~g} \mathrm{ha}^{-1}$ e lactofen a $72 \mathrm{~g} \mathrm{ha}^{-1}$, todos estes utilizados em associação com o herbicida glifosato a $960 \mathrm{~g} \mathrm{ha}^{-1}$. Também foram incluídas três testemunhas, sendo aplicação única do herbicida glifosato a $960 \mathrm{~g} \mathrm{ha}^{-1}$, aplicação sequencial do glifosato a 960/960 $\mathrm{g} \mathrm{ha}^{-1} \mathrm{e}$ testemunha geral sem capina. Os resultados demonstraram que apenas a aplicação única do herbicida glifosato a $960 \mathrm{~g} \mathrm{ha}^{-1}$ e sua associação com as menores doses de cloransulam-metil (10 $\left.\mathrm{g} \mathrm{ha}^{-1}\right)$ e clorimurom-etílico $\left(2,5 \mathrm{~g} \mathrm{ha}^{-1}\right)$ não proporcionaram controle efetivo da trapoeraba. Todos os tratamentos herbicidas não influenciaram na produtividade da soja $\mathrm{RR}^{\circledR}$, embora a associação do glifosato com a maior dose de clorimurom-etílico $\left(10 \mathrm{~g} \mathrm{ha}^{-1}\right)$ tenha proporcionado redução significativa no peso de 1000 grãos de soja.

Palavras-chaves: soja transgênica, fitotoxicidade, seletividade, planta daninha

Abstract - This research aimed to evaluate the selectivity and efficacy of glyphosate tank mixture with different post-emergent herbicides on controlling Commelina benghalensis in soybean $\mathrm{RR}^{\circledR}$ crop. The experiment was conducted in field conditions using complete randomized block design, with four replications, where the treatments were four rates of cloransulam-methyl herbicide $\left(10,20,30\right.$ and $\left.40 \mathrm{~g} \mathrm{ha}^{-1}\right)$, four rates of chlorimuron-ethyl herbicide $(2.5 ; 5.0 ; 7.5$ and $10 \mathrm{~g} \mathrm{ha}^{-1}$ ), imazethapyr at $100 \mathrm{~g} \mathrm{ha}^{-1}$ and lactofen at $72 \mathrm{~g} \mathrm{ha}^{-1}$, all in association with glyphosate herbicide at $960 \mathrm{~g} \mathrm{ha}^{-1}$. It was also included three controls, characterized by an isolated rate of glyphosate at $960 \mathrm{~g} \mathrm{ha}^{-1}$, a sequential application of glyphosate at 960/960 g ae.ha ${ }^{-1}$ besides a check without weeds handle control. The results obtained showed that only the unique application of glyphosate herbicide at $960 \mathrm{~g} \mathrm{ha}^{-1}$ and its association with the lower rate of cloransulam-methyl $\left(10 \mathrm{~g} \mathrm{ha}^{-1}\right)$ or chlorimuron-ethyl $\left(2.5 \mathrm{~g} \mathrm{ha}^{-1}\right)$ did not promote effective $C$.

\footnotetext{
${ }^{1}$ Recebido para publicação em 11/06/2012 e aceito em 26/01/2013.

${ }^{2}$ Professor na Universidade Federal de Mato Grosso - Campus Universitário do Araguaia, Barra do Garças, MT, Brasil. Email: <sidneimarchi.ufmt@gmail.com>. (Autor para correspondência).

${ }^{3}$ Eng. Agr. Syngenta Proteção de Cultivos Ltda., Avenida das Nações Unidas, 18001 - 2o andar, 04795900 São Paulo, SP, Brasil.

${ }^{4}$ Eng. Agr. Agro Norte Pesquisa \& Sementes Ltda. Rod. BR 163 km 841, Cx Postal 405, 78550-970, Sinop, MT, Brasil.
} 
benghalensis control. All chemical treatments did not influence soybean $\mathrm{RR}^{\circledR}$ yield, although the association of glyphosate and the higher rate of chlorimuron-ethyl (10 $\left.\mathrm{g} \mathrm{ha}^{-1}\right)$ have promoted significant reduction in 1000 soybean grains weight.

Keywords: transgenic soybean, phytotoxicity, selectivity, weed

\section{Introdução}

$\mathrm{O}$ advento das culturas geneticamente modificadas, especificamente quanto à resistência ao herbicida glifosato, gerou profundas modificações no controle químico de plantas daninhas (Gazziero et al., 2006). Apesar dos diversos benefícios que a tecnologia proporciona, problemas decorrentes de seu uso repetitivo têm sido informalmente notados e relatados por diversos agricultores. $\mathrm{O}$ controle insatisfatório de algumas espécies de plantas daninhas com esse herbicida aponta indícios de que, a associações com outros ingredientes ativos pode contribuir no manejo de espécies resistentes (Monquero et al., 2001; Carvalho et al., 2003). Uma vez que, resultados de pesquisas têm demonstrado que o uso combinado de glifosato com herbicidas aplicados no manejo de plantas daninhas em soja convencional aumenta o espectro e a eficácia de controle de plantas consideradas mais tolerantes à ação do glifosato isolado (Gonzini et al., 1999; Kranz et al., 2001; Monquero et al., 2001; Vidrine et al., 2002; Norsworthy e Grey, 2004; Procópio et al., 2007; Ramires, et al., 2010).

Espécies de plantas daninhas como corda-de-viola (Ipomoea grandifolia), ervaquente (Spermacoce latifolia), agriãozinho (Synedrellopsis grisebachii), erva-de-touro (Tridax procumbens) e especialmente trapoeraba (Commelina benghalensis) vêm sendo selecionadas devido a aplicações sucessivas de glifosato nas áreas agrícolas do cerrado, resultando em considerável aumento no nível populacional destas espécies. De acordo com Johnson et al. (2002), as falhas no controle de determinadas plantas daninhas pelo uso do glifosato têm levado agricultores a aplicar outros herbicidas em associação, mesmo em soja $\mathrm{RR}^{\circledR}$.
Entre as alternativas para incrementar a eficiência no controle de espécies que apresentam certa tolerância ao glifosato, especialmente em áreas com altas infestações, destacam-se os herbicidas cloransulam-metil, clorimurom-etílico e imazetapir que, além de complementarem a eficiência do glifosato, também possuem relativa atividade residual no solo (Valente e Cavazzana, 2000; Carvalho et al., 2003; Roman, 2002).

Desta forma, o objetivo deste trabalho foi avaliar a seletividade e a eficácia da associação do glifosato com herbicidas pósemergentes no controle de trapoeraba na cultura da soja Roundup Ready ${ }^{\circledR}$.

\section{Material e Métodos}

O experimento foi instalado na Fazenda Chão Nativo localizada no município de Sinop/MT em latitude de $11^{\circ} 48^{\prime} 38,89^{\prime}$ 'S e

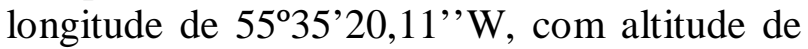
$371 \mathrm{~m}$ acima do nível do mar, utilizando-se a variedade de soja transgênica P98Y70 por se tratar de uma variedade especialmente desenvolvida para os Estados do Centro-Oeste brasileiro. Foi adotado o espaçamento de 55 $\mathrm{cm}$ entre linhas e distribuídas 13 sementes por metro linear no sulco de plantio, obtendo um stand final de aproximadamente 235.000 plantas ha ${ }^{-1}$.

Foi adotado o delineamento experimental em blocos ao acaso, quatro repetições, com os tratamentos experimentais constituídos de quatro doses do herbicida cloransulam-metil (10, 20, 30 e $\left.40 \mathrm{~g} \mathrm{ha}^{-1}\right)$, quatro doses do herbicida clorimurom-etílico $\left(2,5,5,0,7,5\right.$ e $\left.10,0 \mathrm{~g} \mathrm{ha}^{-1}\right)$, imazetapir a $100 \mathrm{~g}$ $\mathrm{ha}^{-1}$ e lactofen a $72 \mathrm{~g} \mathrm{ha}^{-1}$, todos estes utilizados em associação com o herbicida glifosato a $960 \mathrm{~g} \mathrm{ha}^{-1}$. Também foram incluídas três testemunhas, sendo aplicação única do 
herbicida glifosato a $960 \mathrm{~g} \mathrm{ha}^{-1}$, aplicação sequencial do glifosato a 960/ $960 \mathrm{~g} \mathrm{ha}^{-1}$ (intervalo de 21 dias) e testemunha geral sem capina. As parcelas foram constituídas de $8 \mathrm{~m}$ de comprimento e $5 \mathrm{~m}$ de largura, sendo a largura dividida em $3 \mathrm{~m}$ de faixa aplicada e $2 \mathrm{~m}$ de testemunha lateral. Os $24 \mathrm{~m}^{2}$ centrais da faixa aplicada foram considerados com área útil $\mathrm{e}$ onde foram realizadas todas as avaliações.

A aplicação dos herbicidas foi realizada em pós-emergência inicial quando a soja encontrava-se em estádio V2-V3 e a população de trapoeraba (356 plantas $\mathrm{m}^{-2}$ ) em estádio de 2-3 folhas verdadeiras. A pulverização foi realizada com auxílio de um pulverizador costal pressurizado a $\mathrm{CO}_{2}$ equipado com barra disposta a $50 \mathrm{~cm}$ de altura em relação ao nível do solo, contendo seis pontas tipo leque Hypro 100.015, espaçadas $50 \mathrm{~cm}$ entre si, com pressão constante de 1,5 bar e volume de calda equivalente a $130 \mathrm{~L} \mathrm{ha}^{-1}$. As condições climáticas nas aplicações foram semelhantes, com temperatura entre 29,1 e $31,5^{\circ} \mathrm{C}$, umidade relativa do ar entre 63,3 e $73,4 \%$, solo úmido e vento com velocidade de aproximadamente 2,9 $\mathrm{km} \mathrm{h}^{-1}$.

A eficiência dos tratamentos no controle da trapoeraba foi avaliada aos 7, 14, 21, 30 e 42 dias após a aplicação dos tratamentos (DAAT). As associações de herbicidas também foram avaliadas aos 30 DAAT quanto ao efeito de controle residual representado pela supressão no desenvolvimento das plântulas de trapoeraba que emergiram após a pulverização dos tratamentos. A biomassa seca da trapoeraba foi determinada aos 42 DDAT, onde as plantas não controladas e aquelas que emergiram após as aplicações dos herbicidas foram cortadas rente ao solo, embaladas em sacos de papel e secas em estufa de circulação forçada de ar a $60^{\circ} \mathrm{C}$ por 36 horas, quando foi determinada a massa seca da parte aérea por pesagem em balança de precisão. Após a obtenção da biomassa seca foi possível calcular a porcentagem de redução total de plantas de trapoeraba proporcionada por cada tratamento utilizando-se a fórmula $\mathrm{RT}(\%)=\left[\left(\mathrm{BST}_{\mathrm{es}}-\right.\right.$ $\left.\left.\mathrm{BST}_{\mathrm{rt}}\right) \times 100\right] / \mathrm{BST}_{\mathrm{es}}$ onde RT = redução total $\mathrm{BSt}_{\mathrm{es}}=$ biomassa seca de plantas na testemunha e $\mathrm{BST}_{\mathrm{rt}}=$ biomassa seca de plantas no respectivo tratamento, com os valores expressos em porcentagem.

A fitotoxicidade proporcionada pelos tratamentos herbicidas foi avaliada por meio de observação visual, com atribuição de notas de acordo com os sintomas apresentados pelas plantas aos 7, 14, 21 e 30 dias após a aplicação. As notas de controle da trapoeraba e fitotoxicidade na soja foram atribuídas com base na escala em porcentagem de fitotoxicidade proposta por Frans et al. (1986), onde zero representa ausência total de fitotoxicidade e $100 \%$ a morte total das plantas cultivadas. No final, as plantas presentes nos cinco metros das duas linhas centrais da faixa tratada de cada parcela foram manualmente colhidas e debulhadas para determinação da produtividade e do peso de 1000 grãos da soja.

Todos os resultados obtidos foram submetidos à análise de variância pelo teste $\mathrm{F}$ e as médias dos tratamentos comparadas pelo teste de Scott \& Knott (1974) a 5\% de probabilidade.

\section{Resultados e Discussão}

Na Tabela 1 é possível observar que, exceto para a associação entre glifosato e lactofen, a porcentagem de controle da trapoeraba evoluiu lentamente e níveis considerados como aceitáveis (acima de 80\%) foram observados somente nas avaliações realizadas aos 21 dias após a aplicação do tratamento (DAAT). A avaliação realizada aos 21 DAAT também revelou que a adição dos herbicidas cloransulam-metil nas doses de 20 , 30 e $40 \mathrm{~g} \mathrm{ha}^{-1}$, clorimurom-etílico nas doses de 2,$5 ; 5,0 ; 7,5$ e $10 \mathrm{~g} \mathrm{ha}^{-1}$, imazetapir a $100 \mathrm{~g} \mathrm{ha}^{-1}$ e lactofen a $75 \mathrm{~g} \mathrm{ha}^{-1}$ na associação proporcionaram níveis de controle de trapoeraba significativamente superiores àquele 
observado quando o glifosato foi utilizado isoladamente a $960 \mathrm{~g} \mathrm{ha}^{-1}$.

Aos 30 DAAT nota-se que o nível de controle da trapoeraba evoluiu rapidamente para $92,5 \%$ no tratamento em que foi realizada a segunda aplicação do herbicida glifosato. Além disso, a última avaliação de controle realizada aos 42 dias após a aplicação revelou que somente a aplicação única do glifosato a $960 \mathrm{~g} \mathrm{ha}^{-1}$ e sua associação com as menores doses de cloransulam-metil (10 $\left.\mathrm{g} \mathrm{ha}^{-1}\right)$ ou clorimurom-etílico $\left(2,5 \mathrm{~g} \mathrm{ha}^{-1}\right)$ não foram eficientes no controle da trapoeraba. Todos os demais herbicidas e suas respectivas doses utilizadas em associação com glifosato a $960 \mathrm{~g}$ $\mathrm{ha}^{-1}$ foram eficientes e significativamente semelhantes à aplicação sequencial do glifosato (960/ $\left.960 \mathrm{~g} \mathrm{ha}^{-1}\right)$ no controle da trapoeraba (Tabela 1).

Tabela 1. Valores médios de controle sobre plantas de trapoeraba (Commelina benghalensis) proporcionados pelas diferentes associações herbicidas. Sinop, MT. 2010.

\begin{tabular}{|c|c|c|c|c|c|c|}
\hline \multirow[t]{2}{*}{ Associação herbicida } & \multirow{2}{*}{$\begin{array}{l}\text { Dose } \\
\left(\mathrm{g} \mathrm{ha}^{-1}\right)\end{array}$} & \multicolumn{5}{|c|}{ Controle $(\%)-$ DAAT $^{\mathbb{I}}$} \\
\hline & & 7 & 14 & 21 & 30 & 42 \\
\hline 01. glifosato + cloransulam & $960+10$ & $20,0 \mathrm{~b}$ & $27,5 \mathrm{c}$ & $37,5 \mathrm{~d}$ & $58,8 \mathrm{~b}$ & $68,8 \mathrm{~b}$ \\
\hline 02. glifosato + cloransulam & $960+20$ & $15,0 \mathrm{~b}$ & $25,0 \mathrm{c}$ & $65,0 \mathrm{~b}$ & $71,7 \mathrm{a}$ & 93,7 a \\
\hline 03. glifosato + cloransulam & $960+30$ & $17,5 \mathrm{~b}$ & $47,5 \mathrm{~b}$ & $81,2 \mathrm{a}$ & $94,5 \mathrm{a}$ & 96,0 a \\
\hline 04. glifosato + cloransulam & $960+40$ & $20,0 \mathrm{~b}$ & $62,5 \mathrm{~b}$ & 82,6 a & $96,6 \mathrm{a}$ & $97,3 \mathrm{a}$ \\
\hline 05. glifosato + clorimurom & $960+2,5$ & $15,3 \mathrm{~b}$ & $36,3 \mathrm{c}$ & $53,8 \mathrm{c}$ & $57,5 \mathrm{~b}$ & $58,8 \mathrm{c}$ \\
\hline 06. glifosato + clorimurom & $960+5,0$ & $21,2 \mathrm{~b}$ & $40,0 \mathrm{c}$ & $71,3 \mathrm{~b}$ & 86,2 a & $90,7 \mathrm{a}$ \\
\hline 07. glifosato + clorimurom & $960+7,5$ & $22,5 \mathrm{~b}$ & $41,3 \mathrm{c}$ & $73,7 \mathrm{~b}$ & $87,5 \mathrm{a}$ & $96,4 \mathrm{a}$ \\
\hline 08. glifosato + clorimurom & $960+10,0$ & $25,0 \mathrm{~b}$ & $53,8 \mathrm{~b}$ & $75,0 \mathrm{~b}$ & 93,3 a & 95,9 a \\
\hline 09. glifosato + imazetapir & $960+100$ & $18,8 \mathrm{~b}$ & $51,2 \mathrm{~b}$ & 86,3 a & 96,5 a & $97,2 \mathrm{a}$ \\
\hline 10. glifosato + lactofen & $960+72$ & $73,8 \mathrm{a}$ & $87,5 \mathrm{a}$ & $94,5 \mathrm{a}$ & $97,5 \mathrm{a}$ & $97,5 \mathrm{a}$ \\
\hline 11. glifosato & 960 & $8,8 \mathrm{c}$ & $18,8 \mathrm{~d}$ & $36,2 \mathrm{~d}$ & $51,1 \mathrm{~b}$ & $67,7 \mathrm{~b}$ \\
\hline 12. glifosato / glifosato 12 & $960 / 960$ & $12,5 \mathrm{c}$ & $30,0 \mathrm{c}$ & $40,0 \mathrm{~d}$ & $92,5 \mathrm{a}$ & $97,3 \mathrm{a}$ \\
\hline 13. Testemunha & ---- & $0,0 \mathrm{~d}$ & 0,0 e & $0,0 \mathrm{e}$ & $0,0 \mathrm{c}$ & $0,0 \mathrm{~d}$ \\
\hline F Tratamento & & $25,20 *$ & $21,48 *$ & $27,68^{*}$ & $17,33^{*}$ & $159,22 *$ \\
\hline F Bloco & & $0,94^{\mathrm{NS}}$ & $2,10^{\mathrm{NS}}$ & $1,19^{\mathrm{NS}}$ & $1,08^{\mathrm{NS}}$ & $1,25^{\mathrm{NS}}$ \\
\hline C.V. $(\%)$ & & 32,80 & 22,55 & 16,49 & 17,86 & 5,51 \\
\hline
\end{tabular}

Ramires et al. (2010), ao estudarem o controle de Euphorbia heterophylla e Ipomoea grandifolia com a utilização de glifosato isolado ou em associação com latifolicidas na cultura da soja $R R^{\circledR}$, verificaram aumentos na eficácia em relação ao glifosato aplicado isoladamente a $480 \mathrm{~g} \mathrm{ha}^{-1}$, quando este herbicida foi utilizado com herbicidas inibidores de protox. Os mesmos autores mencionaram ainda que misturas contendo glifosato a $960 \mathrm{~g} \mathrm{ha}^{-1} \mathrm{e}$ outros herbicidas pós- emergentes (cloransulam-methyl, clorimuromethyl, imazetapir, lactofen, fomesafen, flumiclorac-pentyl e bentazon) proporcionaram incrementos da eficácia de controle, independentemente se as planta daninhas estavam no estádio de 2 a 3 ou 4 a 6 folhas.

Especialmente em relação à trapoeraba, resultados semelhantes foram obtidos por Monquero et al. (2001) e Procópio et al. (2007), os quais observaram incrementos no nível de controle da espécie $C$. benghalensis 
quando o glifosato foi utilizado em associação com clorimurom-etílico ou com imazetapir.

$\mathrm{Na}$ Tabela 2 é possível notar que algumas associações entre glifosato e os herbicidas pós-emergentes proporcionaram significativos efeitos residuais e, consequente, supressão das plantas de trapoeraba que emergiram após a aplicação. Destaca-se que o melhor efeito residual foi proporcionado pela associação entre glifosato a $960 \mathrm{~g} \mathrm{ha}^{-1} \mathrm{e}$ imazetapir na dose de $100 \mathrm{~g} \mathrm{ha}^{-1}$, onde observou-se $77,5 \%$ de redução na quantidade de plântulas de trapoeraba. Também foi observado efeito residual da associação entre o glifosato e as maiores doses de cloransulammetil (40 $\left.\mathrm{g} \mathrm{ha}^{-1}\right)$ e clorimurom-etílico (10 $\mathrm{g} \mathrm{ha}^{-}$ $\left.{ }^{1}\right)$, uma vez que ambas proporcionaram aproximadamente $50 \%$ de redução das plântulas de trapoeraba. Porém, salienta-se que estas duas associações foram significativamente inferiores à associação entre glifosato e imazetapir.

Tabela 2. Supressão de plântulas e produção de biomassa seca de trapoeraba (Commelina benghalensis) observada nas diferentes associações herbicidas. Sinop, MT. 2010.

\begin{tabular}{|c|c|c|c|c|}
\hline Associação herbicida & $\begin{array}{c}\text { Dose } \\
\left(\mathrm{g} \mathrm{ha}^{-1}\right)\end{array}$ & $\begin{array}{c}\begin{array}{c}\text { Supressão } \\
\text { plântulas }(\%)^{11}\end{array} \\
30 \mathrm{DAAT}^{\underline{3}} \\
\end{array}$ & $\begin{array}{c}\begin{array}{c}\text { Biomassa seca } \\
\left(\mathrm{g} \cdot \mathrm{m}^{-2}\right)^{\backslash 1}\end{array} \\
42 \text { DAAT }\end{array}$ & $\begin{array}{c}\begin{array}{c}\text { Redução total } \\
(\%)^{12}\end{array} \\
42 \text { DAAT } \\
\end{array}$ \\
\hline 01. glifosato + cloransulam & $960+10$ & $2,02(6,3) \mathrm{e}$ & $4,66(22,9) \mathrm{c}$ & 71,7 \\
\hline 02. glifosato + cloransulam & $960+20$ & $4,01(20,0) \mathrm{d}$ & $3,06(12,2) \mathrm{c}$ & 84,9 \\
\hline 03. glifosato + cloransulam & $960+30$ & $4,83(22,5) \mathrm{c}$ & $3,51(8,7) \mathrm{c}$ & 89,2 \\
\hline 04. glifosato + cloransulam & $960+40$ & $7,35(53,7) b$ & $2,69(6,4) c$ & 92,1 \\
\hline 05. glifosato + clorimurom & $960+2,5$ & $1,00(0,0) \mathrm{e}$ & $4,77(24,0) \mathrm{c}$ & 70,3 \\
\hline 06. glifosato + clorimurom & $960+5,0$ & $3,52(12,5) \mathrm{d}$ & $4,43(21,8) \mathrm{c}$ & 73,0 \\
\hline 07. glifosato + clorimurom & $960+7,5$ & $5,28(28,9) \mathrm{c}$ & $3,45(11,6) \mathrm{c}$ & 85,6 \\
\hline 08. glifosato + clorimurom & $960+10,0$ & $7,03(48,8) b$ & $3,30(10,4) \mathrm{c}$ & 87,1 \\
\hline 09. glifosato + imazetapir & $960+100$ & $8,85(77,5) \mathrm{a}$ & $2,48(5,5) \mathrm{c}$ & 93,2 \\
\hline 10. glifosato + lactofen & $960+72$ & $(1,00) 0,0 \mathrm{e}$ & $2,62(6,7) c$ & 91,7 \\
\hline 11. glifosato & 960 & $1,00(0,0) \mathrm{e}$ & $8,87(80,8) b$ & ---- \\
\hline 12. glifosato + glifosato ${ }^{4}$ & $960+960$ & $1,00(0,0) \mathrm{e}$ & $2,75(7,8) \mathrm{c}$ & 90,3 \\
\hline 13. Testemunha & ---- & $1,00(0,0) \mathrm{e}$ & $13,18(185,4) \mathrm{a}$ & ---- \\
\hline F Tratamento & & $33,66^{*}$ & $13,58^{*}$ & \\
\hline F Bloco & & $5,70^{*}$ & $1,46^{\mathrm{NS}}$ & \\
\hline C.V. (\%) & & 26,21 & 36,37 & \\
\hline
\end{tabular}

${ }^{\text {NS }}=$ Não significativo; * Significativo ao nível de 5\% de probabilidade. Médias seguidas de mesma letra na coluna não diferem significativamente entre si pelo teste de Scott-Knott a 5\% de probabilidade.

${ }^{1}$ Médias transformadas em raiz $(X+1,0)$. Médias originais entre parêntesis; ${ }^{12}$ Em relação à testemunha; $\stackrel{13}{ }$ DAAT $=$ dias após a aplicação do tratamento; ${ }^{14}$ Intervalo de 21 dias entre aplicações.

Os herbicidas cloransulam-metílico, clorimurom-etílico e imazetapir apresentam ação em aplicações em pós-emergência, porém podem também oferecer atividade residual no solo, podendo ser benéfico quando se pretende fazer apenas uma aplicação em pós-emergência na cultura da soja RR $^{\circledR}$ (Carvalho et al., 2003; Villa et al., 2006; Oliveira Neto et al., 2013). Isso porque o glifosato não apresenta ação no solo, e a realização de apenas uma aplicação em pós-emergência pode causar, dependendo do tempo de fechamento do dossel, reinfestação de plantas daninhas, ocasionando problemas na colheita mecanizada (Procópio et al., 2007).

Os controles juntamente com os efeitos residuais sobre as plantas de trapoeraba proporcionados pelas associações entre $\mathrm{O}$ 
glifosato e os demais herbicidas explicam a produção de biomassa seca da trapoeraba observada aos 42 DAAT. Nota-se ainda na Tabela 2 que todas as associações foram significativamente superiores à aplicação única do glifosato a $960 \mathrm{~g} \mathrm{ha}^{-1}$, porém semelhantes à aplicação sequencial do mesmo herbicida quanto à produção de biomassa seca. Destacase que, embora significativamente semelhante, reduções efetivas e acima de $90 \%$ na produção de biomassa seca de trapoeraba foram observadas somente nas associações do glifosato com a maior dose de cloransulammetil (40 $\left.\mathrm{g} \mathrm{ha}^{-1}\right)$ e imazetapir, na dose de $100 \mathrm{~g}$ $\mathrm{ha}^{-1}$.

Resultados semelhantes foram obtidos por Monquero et al. (2001), os quais verificaram que os tratamentos mais eficazes na redução percentual da biomassa seca de trapoeraba foram glifosato, aplicado isoladamente, e a mistura deste herbicida com carfentrazone, flumioxazin, sulfentrazone, clorimurom-etílico e bentazon. Salienta-se que todos os herbicidas utilizados neste estudo, de acordo com Rodrigues \& Almeida (1998), são recomendados no controle pós-emergente de trapoeraba.

$\mathrm{Na}$ Tabela 3 nota-se que a associação do glifosato (960 $\mathrm{g} \mathrm{ha}^{-1}$ ) com clorimurometílico nas doses de 7,5 e $10 \mathrm{~g} \mathrm{ha}^{-1}$, imazetapir $\left(100 \mathrm{~g} \mathrm{ha}^{-1}\right)$ e lactofen $\left(75 \mathrm{~g} \mathrm{ha}^{-1}\right)$ proporcionaram consideráveis sintomas visuais de fitotoxicidade em plantas de soja RR P98Y70 aos 7 DAAT. A associação da mesma dose de glifosato com as diferentes doses de cloransulam-metil também proporcionaram sintomas visuais de fitotoxicidade em plantas de soja, porém os valores observados sempre foram inferiores a $10 \%$.

Tabela 3. Evolução da fitotoxicidade, produtividade e peso médio de 1000 grãos observados nas associações entre glifosato e diferentes herbicidas aplicados na pós-emergência da soja RR. Sinop, MT. 2010.

\begin{tabular}{|c|c|c|c|c|c|c|c|}
\hline \multirow{2}{*}{ Associação herbicida } & \multirow{2}{*}{$\begin{array}{c}\text { Dose } \\
\left(\mathrm{g} \mathrm{ha}^{-1}\right)\end{array}$} & \multicolumn{4}{|c|}{ Fitotoxicidade (\%) - DAAT 1} & \multirow{2}{*}{$\begin{array}{c}\text { Produtividade } \\
\left(\mathrm{kg} \mathrm{ha}^{-1}\right)\end{array}$} & \multirow{2}{*}{$\begin{array}{c}\text { Peso } 1000 \text { grãos } \\
\text { (g) }\end{array}$} \\
\hline & & 7 & 14 & 21 & 30 & & \\
\hline 01. glifosato + cloransulam & $960+10$ & $8,7 \mathrm{~b}$ & 2,5 & 0,0 & 0,0 & $3364,1 \mathrm{a}$ & 149,6 a \\
\hline 02. glifosato + cloransulam & $960+20$ & $8,8 \mathrm{~b}$ & 2,5 & 0,0 & 0,0 & 3544,5 a & $155,1 \mathrm{a}$ \\
\hline 03. glifosato + cloransulam & $960+30$ & $6,2 \mathrm{~b}$ & 2,6 & 0,0 & 0,0 & 3588,2 a & $153,8 \mathrm{a}$ \\
\hline 04. glifosato + cloransulam & $960+40$ & $10,0 \mathrm{~b}$ & 5,0 & 0,0 & 0,0 & $3646,4 \mathrm{a}$ & $153,7 \mathrm{a}$ \\
\hline 05. glifosato + clorimurom & $960+2,5$ & $4,5 \mathrm{c}$ & 0,0 & 0,0 & 0,0 & $3692,3 \mathrm{a}$ & $151,8 \mathrm{a}$ \\
\hline 06. glifosato + clorimurom & $960+5,0$ & $9,5 \mathrm{~b}$ & 2,4 & 0,0 & 0,0 & $3620,9 \mathrm{a}$ & $156,6 \mathrm{a}$ \\
\hline 07. glifosato + clorimurom & $960+7,5$ & $14,5 \mathrm{a}$ & 7,5 & 3,3 & 0,0 & $3560,9 \mathrm{a}$ & 156,2 a \\
\hline 08. glifosato + clorimurom & $960+10,0$ & 20,0 a & 10,0 & 3,7 & 0,0 & $3046,8 \mathrm{a}$ & $139,9 \mathrm{~b}$ \\
\hline 09. glifosato + imazetapir & $960+100$ & $15,0 \mathrm{a}$ & 12,5 & 3,8 & 0,0 & $3658,6 \mathrm{a}$ & $157,4 \mathrm{a}$ \\
\hline 10. glifosato + lactofen & $960+72$ & $22,5 \mathrm{a}$ & 11,3 & 6,3 & 0,0 & $3531,8 \mathrm{a}$ & $152,6 \mathrm{a}$ \\
\hline 11. glifosato & 960 & $0,0 \mathrm{c}$ & 0,0 & 0,0 & 0,0 & $3493,6 \mathrm{a}$ & 153,9 a \\
\hline 12. glifosato + glifosato 2 & $960+960$ & $0,0 \mathrm{c}$ & 0,0 & 0,0 & 0,0 & 3814,1 a & $151,7 \mathrm{a}$ \\
\hline 13. Testemunha & --- & $0,0 \mathrm{c}$ & 0,0 & 0,0 & 0,0 & $2092,3 \mathrm{~b}$ & $140,4 \mathrm{~b}$ \\
\hline F Tratamento & & $5,83^{*}$ & $1,37^{\mathrm{NS}}$ & $1,78^{\mathrm{NS}}$ & $\ldots$ & $4,18^{*}$ & $1,75^{*}$ \\
\hline F Bloco & & $9,43^{*}$ & $4,37^{*}$ & $1,60^{\mathrm{NS}}$ & $-\cdots$ & $3,91^{*}$ & $2,09^{\mathrm{NS}}$ \\
\hline C.V. $(\%)$ & & 63,97 & 165,40 & 246,20 & $-\cdots$ & 12,69 & 5,54 \\
\hline
\end{tabular}

${ }^{\mathrm{NS}}=$ Não significativo; * Significativo ao nível de $5 \%$ de probabilidade. Médias seguidas de mesma letra na coluna não diferem estatisticamente entre si pelo teste de Scott-Knott a 5\% de probabilidade.

11 - DAAT = dias após a aplicação do tratamento; ${ }^{12}$ Intervalo de 21 dias entre aplicações.

Todos os sintomas visuais de da cultura, uma vez que apenas a testemunha fitotoxicidade observados nas plantas de soja sem capina apresentou produtividade regrediram rapidamente nas avaliações significativamente inferior aos demais seguintes e efetivamente desapareceram aos 30 tratamentos herbicidas. Cabe salientar que DAAT. Estes sintomas de fitotoxicidade apenas a associação entre os herbicidas também não refletiram em perdas na produção glifosato $\left(960 \mathrm{~g} \mathrm{ha}^{-1}\right)$ e clorimurom-etílico a 10 
g ha ${ }^{-1}$ e a testemunha sem capina proporcionaram reduções significativas no peso médio de 1000 grãos (Tabela 2).

Assim como neste estudo, Procópio et al. (2007) e Maciel et al. (2009) ao estudarem o efeito da associação do glifosato com os herbicidas imazetapir ou clorimurom-etílico, também observaram sintomas visuais de intoxicação iniciais em plantas de soja, principalmente quando o clorimurom-etílico foi utilizado com óleo mineral. Entretanto, ambos os autores comentam que as associações não promoveram reduções significativas de produtividade para os cultivares de soja RR estudados.

\section{Conclusões}

Controles efetivos com consideráveis reduções de biomassa seca de plantas de Commelina benghalensis presentes em altas infestações em lavouras de soja RR podem ser obtidos com apenas uma aplicação de glifosato a $960 \mathrm{~g} \mathrm{ha}^{-1}$ associado aos herbicidas pósemergentes cloransulam-metil, clorimurometílico, imazetapir ou lactofen, sem que ocorra perda significativa de produtividade.

\section{Referências}

CARVALHO, F.T. et al. Manejo químico das plantas daninhas Euphorbia heterophylla e Bidens pilosa em sistema de plantio direto da cultura de soja. Planta Daninha, v.21, n.1, p.145-150, 2003.

FRANS, R. et al. Experimental design and techniques for measuring and analyzing plant responses to weed control practices. In: CAMPER, N. D. (Ed.) Research methods in weed science, Third Edition USA SOUTHERN WEED SCIENCE SOCIETY, 1986. p.29-46.

GAZZIERO, D.L.P. et al. Deposição de glyphosate aplicado para controle de plantas daninhas em soja transgênica. Planta Daninha, v.24, n.1, p.173-181, 2006.
GONZINI, L.C.; HART, E.S.; WAX, L.M. Herbicide combination for weed management in glyphosate-resistant soybean. Weed Technology, v.13, n.2, p.354-360, 1999.

KRANZ, R.F. et al. Influence of weeds competition and herbicides on glyphosate resistant soybean (Glycine max). Weed Technology, v.15, n.3, p.530-534, 2001.

JOHNSON, B.F. et al. Herbicide effects on visible injury, leaf area, and yield of glyphosate-resistant soybean (Glycine max). Weed Technology, v.16, n.3, p.554-566, 2002.

MACIEL, C.D.G. et al. Seletividade de cultivares de soja RR submetidos a misturas em tanque de glyphosate + chlorimuron-ethyl associadas a óleo mineral e inseticidas. Planta Daninha, v.27, n.4, p.755-768. 2009.

MONQUERO, P.A.; CHRISTOFOLLETI, P.J.; SANTOS, C.T.D. Glyphosate em mistura com herbicidas alternativos para o manejo de plantas daninhas. Planta Daninha, v.19, n.3, p.375-380, 2001.

NORSWORTHY, J.K.; GREY, T.L. Addition of nonionic surfactant to glyphosate plus chlorimuron. Weed Technology, v.18, n.3, p.588-593, 2004.

OLIVEIRA NETO, A.M. et al. Sistemas de dessecação de manejo com atividade residual no solo para áreas de pousio de inverno infestadas com buva. Comunicata Scientiae, v.4, n.2, p.120-128, 2013.

PROCÓPIO, S.O. et al. Utilização de chlorimuron-ethyl e imazethapyr na cultura da soja Roundup Ready. Planta Daninha, v.25, n.2, p.365-373. 2007.

RAMIRES, A.C. et al. Controle de Euphorbia heterophylla e Ipomoea grandifolia com a utilização de glyphosate isolado ou em associação com latifolicidas. Planta Daninha, v.28, n.3, p.621.629. 2010.

RODRIGUES, B.N.; ALMEIDA F.S. Guia de herbicidas. Londrina: IAPAR, 1998. 648p. 
ROMAN, E.S. Eficácia de herbicidas na dessecação e no controle residual de plantas daninhas no sistema desseque e plante. Revista Brasileira de Herbicidas, v.3, n.1, p.45-49, 2002.

SCOTT, A.; KNOTT, M. Cluster-analysis method for grouping means in analysis of variance. Biometrics, v.30, n.3, p.507-512, 1974.

VALENTE, T.O.; CAVAZZANA, M.A. Efeito residual de chlorimuron-ethyl aplicado em mistura com glyphosate na dessecação de plantas daninhas. Revista Brasileira de Herbicidas, v.1, n.2, p.173-178, 2000.

VIDRINE, R.P.; GRIFFIN, J.L.; BLOUIN, D.C. Evaluation of reduced rates of glyphosate and chlorimuron in glyphosate resistant soybean (Glycine max). Weed Technology, v.16, n.4, p.731-736, 2002.

VILLA, S.C.C. et al. Arroz tolerante a imidazolinonas: controle do arroz-vermelho, fluxo gênico e efeito residual do herbicida em culturas sucessoras não-tolerantes. Planta Daninha, v.24, n.4, p.761-768, 2006. 